\title{
Traumatic Brain Injuries in Internal Medicine Wards: An Emerging Challenge Needing for a Call to Action
}

\author{
Luca Masotti ${ }^{1, ~ *, ~ G i a n n i ~ L o r e n z i n i ~}{ }^{2}$, Irene Chiti ${ }^{3}$, Laura Policardo ${ }^{4}$, Paolo Francesconi ${ }^{4}$, \\ Grazia Panigada ${ }^{3}$, Giancarlo Landini ${ }^{1}$
}

${ }^{1}$ Medicina Interna, Ospedale Santa Maria Nuova, Florence, Italy

${ }^{2}$ Medicina Interna, Ospedale di Borgo San Lorenzo, Florence, Italy

${ }^{3}$ Medicina Interna, Ospedale di Pescia, Pistoia, Italy

${ }^{4}$ Agenzia Regionale di Sanità della Toscana Osservatorio di Epidemiologia, Florence, Italy

Email address:

luca.masotti@tin.it (L. Masotti)

\section{To cite this article:}

Luca Masotti, Gianni Lorenzini, Irene Chiti, Laura Policardo, Paolo Francesconi, GraziaPanigada, Giancarlo Landini. Traumatic Brain Injuries in Internal Medicine Wards: An Emerging Challenge Needing for a Call to Action. American Journal of Internal Medicine. Vol. 3, No. 2, 2015, pp. 50-54. doi: 10.11648/j.ajim.20150302.12

\begin{abstract}
Background: Traumatic brain injuries (TBIs) represent fearing occurrences in clinical practice, due to high mortality and morbidity. Whether the burden of TBIs is increased in Internal Medicine wards remains unclear, therefore the aim of our study was to provide information about it. Materials and Methods: International Classification of Diseases, $9^{\text {th }}$ revision, Clinical Modification (ICD- $9^{\text {th }}$ CM) database referred to patients discharged from Tuscan Hospitals in the 2003, 2008 and 2012 years was analyzed. We searched for codes from 850 to 853.19 and from 854.00 to 854.19 as traumatic intracranial bleedings (TIBs) and codes from 800.00 to 804.99 as traumatic brain injuries without bleedings (TBIWBs) in the first six diagnoses of hospital discharge schedules referred to Internal Medicine wards. Results: Overall, hospital discharges for TBIs reduced over the years. However, hospital discharges for TBIs were 47,2\% increased in Internal Medicine wards from 2003 to 2012. TBIs increased in 3,4\% of patients under 75 years and $93,7 \%$ in patients over 75 years. Internal Medicine ward was the hospital setting in 11,20\% and 16,70\% of TIBs and TIBWBs in 2003 and 24,10\% and 37.50\%in 2012, respectively. In-hospital mortality in TBIs patients was unchanged over the years and was seven-tenfold greater in patients over 75 years compared to patients under 75 years. Conclusion: Admissions for TBIs are dramatically increased over the last 10 years in Internal Medicine wards, especially in patients over 75 years, making these conditions as a real emerging challenge for the Internists.
\end{abstract}

Keywords: Traumatic Brain Injury, Intracranial Bleedings, Epidemiology, Internal Medicine

\section{Introduction}

Traumatic brain injuries (TBIs) are considered between the most feared occurrences in hospital clinical practice due to high mortality and morbidity.

Incidence of overall TBIs is estimated to be around $235 / 100.00$ persons/years and severe TBIs 7-20 person/100.000 per year (1). Incidence and mortality for TBIs increase steadily with age (1). Despite the overall incidence of TBIs seem to be fallen in the last decades, the incidence of TBIs in people over 65 injured in non-trafficrelated circumstances seem to be risen dramatically, being fall the major risk factor in the elderly (2).Mortality associated to TBIs varies according to severity, the mean being around 11\% (1). Functional sequels occur in around of
$40 \%$ of patients with TBIs (3).

TBIs have been traditionally considered to be pertinence of neurologists, neurosurgeons and neuro-intensivists (4). However these conditions have a wide spectrum of severity, ranging from asymptomatic to severe presentation associated to comatose state $(4,5)$. Moreover many aspects of acute and post-acute phases require typically a medical approach, such as blood pressure and glycemic control, management of fever, prevention and treatment of infectious respiratory diseases and respiratory failure, management of antithrombotic drugs, prevention and treatment of venous thromboembolism, management of nutritional disorders and functional rehabilitation $(4,5)$. Therefore it's not surprisingly that a lot of TBIs patients are recovered in medical wards, especially in the case of patients not urgently requiring neurosurgical 
approach or unsuitable for neurosurgical approach. Whether the burden of TBIs is increased in Internal Medicine wards is unclear, but it's common opinion within the Internists that these conditions seem to be much more frequent in their clinical practice in the last decade, especially in older patients. Due to the lack of epidemiological data referred to TBIs admitted and discharged from Internal Medicine wards, the aim of our study was to provide information about it.

\section{Materials and Methods}

The International Classification of Diseases, $9^{\text {th }}$ revision, Clinical Modification (ICD- $9^{\text {th }} \mathrm{CM}$ ) is a worldwide system of assigning codes to diagnoses and procedures associated with hospital utilization and it is used in many Italian regions for this purpose since the first years of the last decade. We performed a retrospective analysis of the ICD $-9^{\text {th }} \mathrm{CM}$ codes of patients discharged from Hospitals of Tuscany in the 2003, 2008 and 2012 years. Tuscany is one of the leading region of Italy, encompassing for around 3.692.000 inhabitants, 12,3\% of them being over 75 years. We searched for all codes encompassing between 850 and 853.19 and between 854.00 and 854.19 as traumatic intracranial bleedings (TIBs) and for codes between 800.00 and 804.99 as traumatic brain injuries without bleedings (TBIWBs). The abovementioned codes were searched within the first six diagnoses of hospital discharge schedules.

\section{Results}

705.089 patients were discharged from Tuscan Hospitals in 2003 (129.349 from Internal Medicine wards, 50.9\% over 75 years), whereas 611.397 were in 2012 (106.866 in Internal Medicine wards, $61.8 \%$ over 75 years). Therefore in the last decade, the overall hospital admissions in Internal Medicine wards of Tuscany were $17,3 \%$ reduced.

Overall, hospital discharges for TBIs reduced over the years, from 9687 in 2003 to 6515 in 2012 . However, hospital discharges for TBIs were $47,2 \%$ increased in Internal Medicine wards (from 1476 in 2003 to 2173 in 2012)(Figure 1). According to age, hospital discharges for TBIs were $3,4 \%$ increased in patients under 75 years, whereas these were $93,7 \%$ increased in patients over 75 years (Figure 2). Internal Medicine ward was the hospital setting of $11,20 \%$ and $16,70 \%$ of TIBs and TIBWBs in 2003 and 24,10\% and $37.50 \%$ in 2012, respectively (Figure 3 ).

In-hospital mortality in patients with TBIs was unchanged over the years, being around $4 \%$, but it was seven-tenfold greater in patients over 75 years compared to patients under 75 years (Figure 4 ).

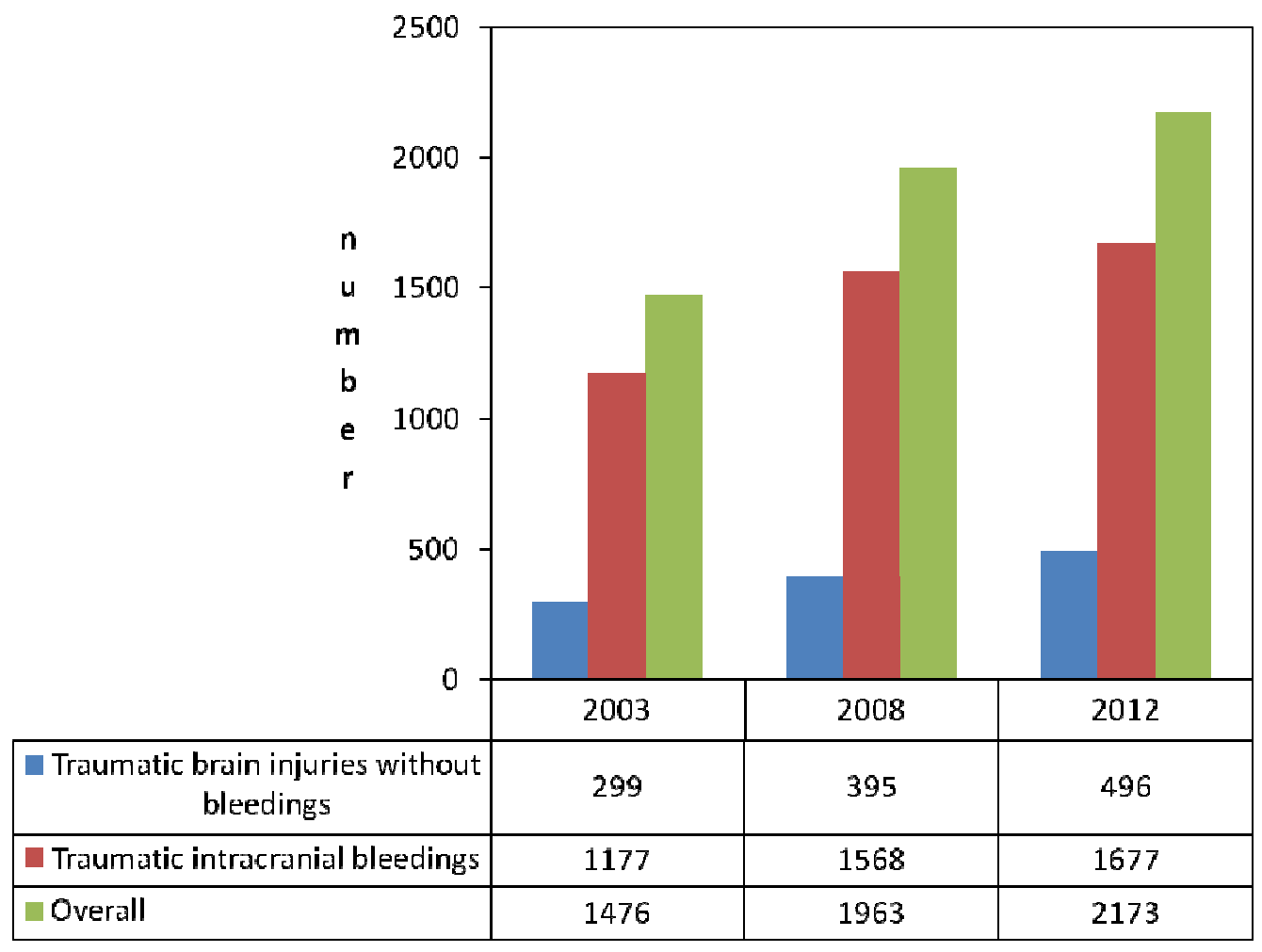

Figure 1. Trend in hospital discharges for traumatic brain injuries in Internal Medicine wards. 


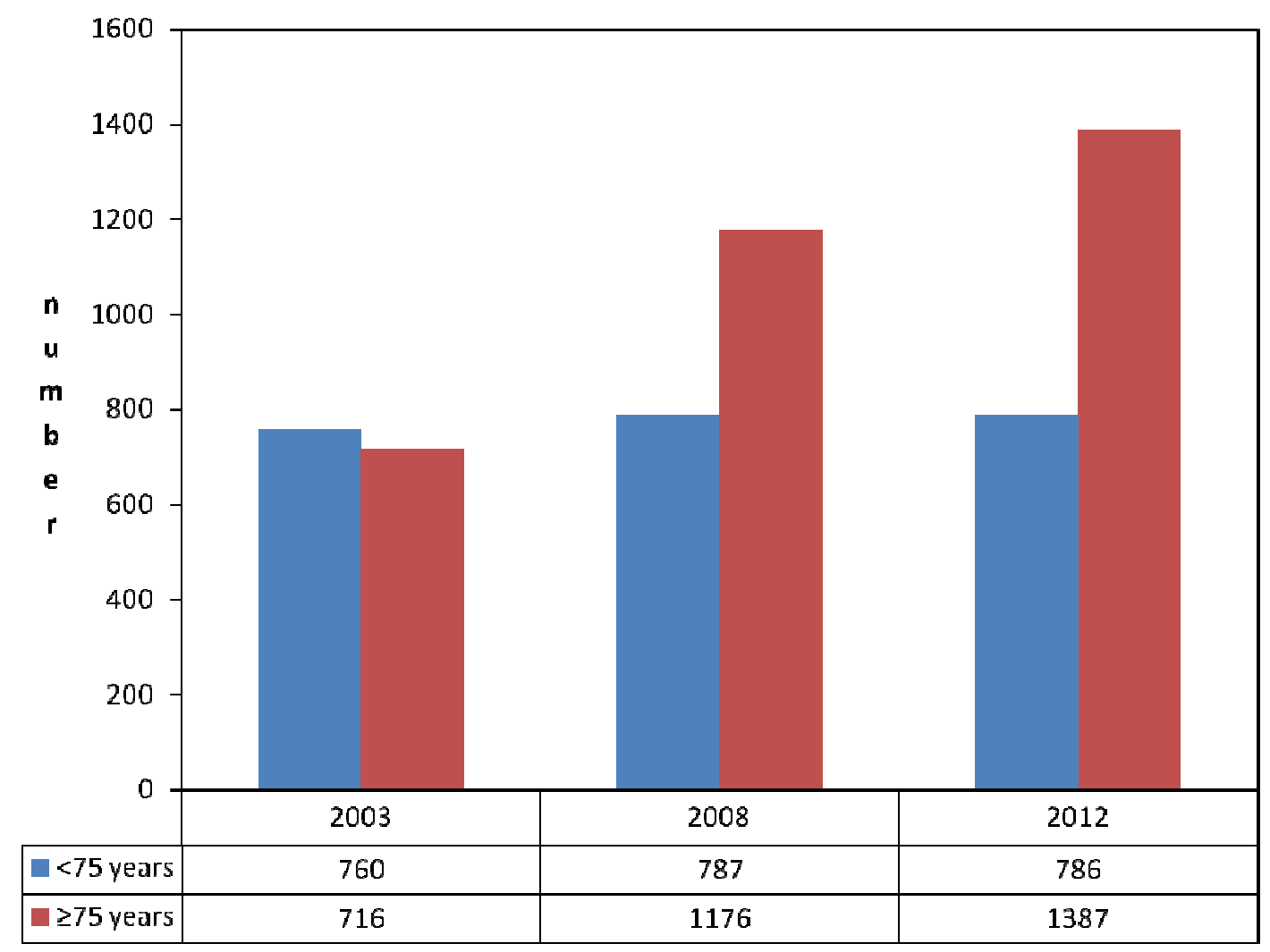

Figure 2. Trend in hospital discharges for traumatic brain injuries over the years according to age.

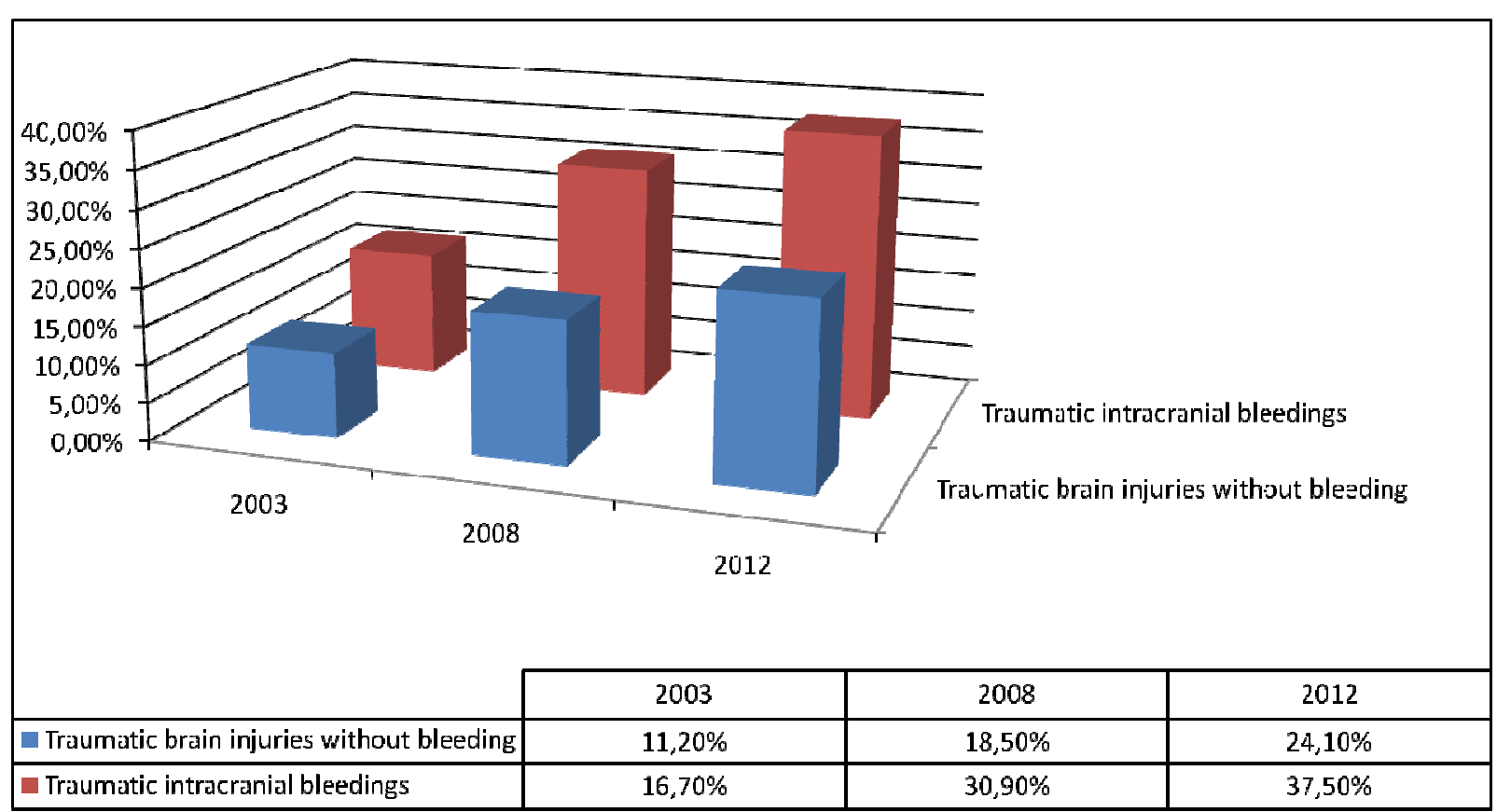

Figure 3. Trend in the burden of Internal Medicine ward as setting for traumatic brain injuries. 


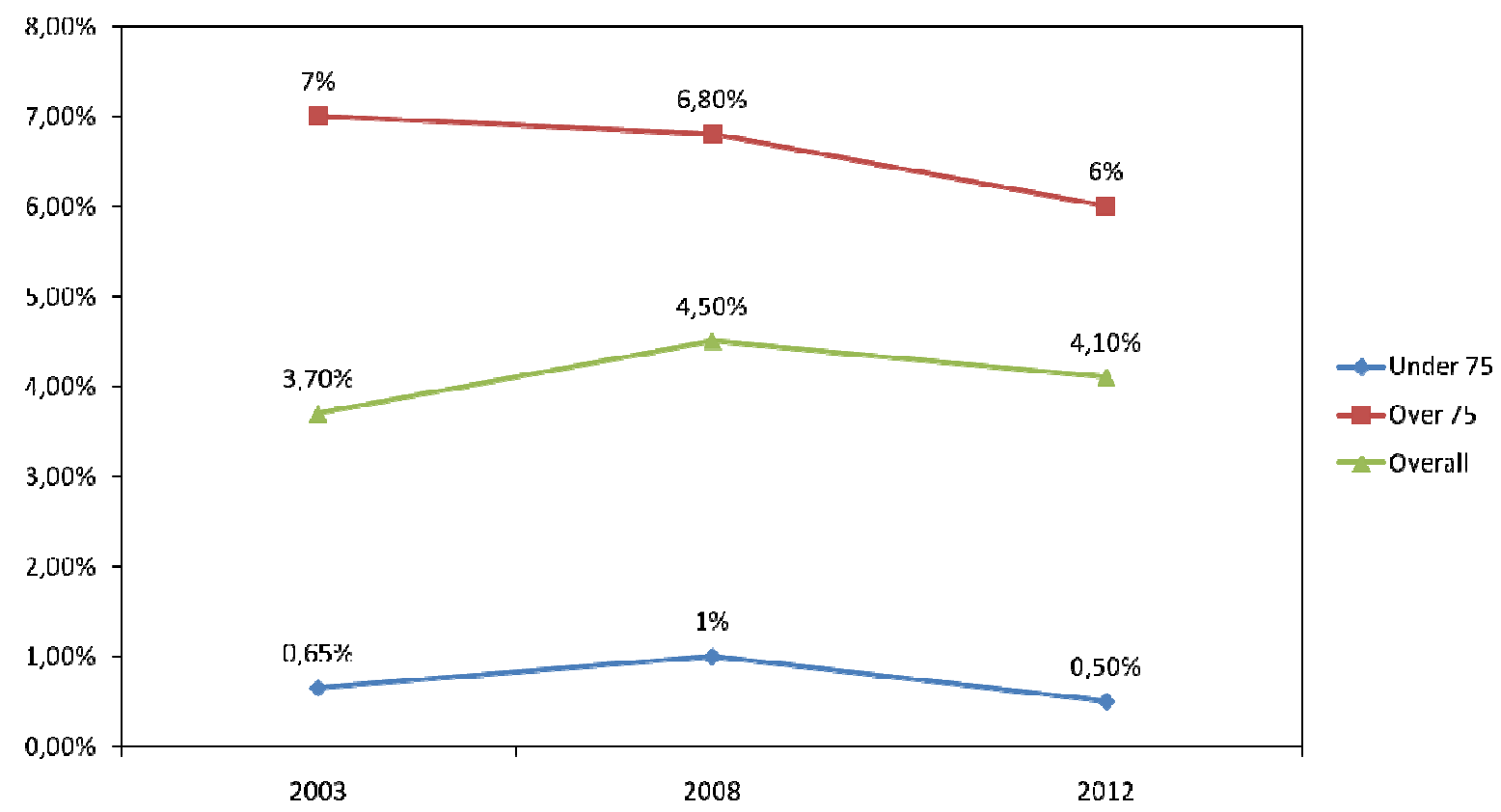

Figure 4. Trend in mortality for traumatic brain injuries in Internal Medicine wards according to age.

\section{Discussion}

TBIs are feared conditions, burdened by high mortality and morbidity, and difficult to manage. Our study confirms the common opinion between Internists about their speculation that TBIs are increased in their wards over the years, especially when considering the very old patients. In the last decade, the overall number of admissions for TBIs reduced in Tuscany as trends reported in literature (6-8), but when considering patients over 75 years the overall hospital admissions for TBIs increased both in numbers (2469 in 2003, 2949 in 2012) and in burden of patients over 75 injured on total patients $(25,4 \%$ in $2003,45.2 \%$ in 2012$)$, our results being overlapping to literature $(1,6-12)$. Concomitantly, the burden of admissions for TBIs in Internal Medicine wards increased and this increasing was totally dependent from that occurred in patients over 75 years. This fact could be interpreted as the result of the general aging of patients admitted in Internal Medicine wards, but on the other hand it could be explained with a major selection of patients. The other than Internal Medicine wards probably select clinically younger and more severe patients and patients who need for surgical approach or intensive care leaving to Internal Medicine older and clinically less severe patients, not requiring urgent neurosurgical or intensive approach or on the other hand highly severe patients unsuitable for advantages from surgical approaches or at highest periprocedural risks. The patients' selection is the cornerstone of the neurosurgical approach both for spontaneous intracerebral hemorrhage and TBIs as recommended from practical guidelines (5). Performance and neurological status other than volume and localization of intracranial bleedings represent the major points to be considered in the selection of the neurosurgical choice. Therefore, older patients who frequently have more frailty and co-morbidity could be excluded from the neurosurgical approach for a poor performance status which may lead to higher anaesthesiological and surgical risk and it's not surprisingly that these patients who need for a holistic approach may become pertinence of the Internists.

\section{Limitations}

We recognize that our study presents limitations. We have used the ICD- $9^{\text {th }} \mathrm{CM}$, coding of which is operator depending. Therefore coding bias could be enclosed, especially when considering the first years from ICD- $9^{\text {th }} \mathrm{CM}$ introduction in Italy, such as the year 2003. However, the potentially bias enclosed should encompass both internal and non internal medicine wards. Moreover, we searched diagnoses between the first six diagnoses of hospital discharge schedule, therefore it's unclear neither if TBIs were the reason of hospital admission nor if patients were firstly admitted in a non internal medicine ward and accordingly transferred in internal medicine wards. Finally, we have enclosed only the patients who were admitted, therefore the numbers could be underestimated due to lack of patients with mild TBIs directly discharged from Emergency Departments or with severe TIBs rapidly deteriorated and died in this setting. At this purpose, it should be remarked that in the last decade many Italian Hospitals have been equipped of Emergency Medicine Units or Brief Observation Units associated to Emergency Departments, therefore many patients could be discharged from Hospitals in the first 48-72 hours without admission in Internal Medicine or other wards. Since hospital discharge schedules are not compiled by physicians working in Emergency Rooms and Brief Observation Units, our study could underestimate the true burden of TBIs. However, this 
potential bias in our study, should regard much more the last years (2008-2012) and therefore could arise the hypothesis that the reduction in prevalence could be not really true or so consistent over the years as resulted from our study and on the other side it could arise the hypothesis that the magnitude of problem in Internal Medicine wards and in the elderly could be much more frequent yet.

\section{Conclusion}

Despite overall reducing, TBIs represent a public health problem and a real emerging challenge in the setting of Internal Medicine wards, especially when considering the patients over 75 years. Literature evidence lacks when referred to TBIs managed in Internal Medicine wards, therefore our study could add information about this topic and call to action. In fact, the Internists are urgently called to give prompt reply to these emerging and challenging conditions in terms of organization and management protocols.

\section{References}

[1] Tagliaferri F, Compagnone C, Korsic M, Servadei F, Kraus J. A systematic review of brain injury epidemiology in Europe. ActaNeurochir (Wien) 2006; 148: 255-68

[2] Pérez K, Novoa AM, Santamariña-Rubio E et al.; Working Group for Study of Injuriesof Spanish Society of Epidemiology. Incidence trends of traumatic spinal cord injury and traumatic brain injury in Spain, 2000-2009. Accid Anal Prev 2012; 46: 37-44.

[3] Selassie AW, Zaloshnja E, Langlois JA, Miller T, Jones P, Steiner C. Incidence of long-term disability following traumatic brain injury hospitalization, UnitedStates, 2003. J Head Trauma Rehabil. 2008 Mar-Apr; 23(2):123-31.
[4] Rusticali B, Villani R; Working Group. Treatment of minor and severe traumatic brain injury. National reference guidelines. Minerva Anestesiol 2008; 74):583-616.

[5] Brain Trauma Foundation; American Association of Neurological Surgeons; Congress of Neurological Surgeons. Guidelines for the management of severe traumatic brain injury. J Neurotrauma. 2007; 24Suppl 1:S1-106.

[6] Andelic N, Anke A, Skandsen T, Sigurdardottir S, Sandhaug $\mathrm{M}$, Ader T, Roe C. Incidence of hospital-admitted severe traumatic brain injury and in-hospital fatality in Norway: a national cohort study. Neuroepidemiology 2012; 38:259-67.

[7] Colantonio A, Croxford R, Farooq S, Laporte A, Coyte PC. Trends in hospitalization associated with traumatic brain injury in a publicly insured population, 1992-2002. J Trauma 2009; 66:179-83.

[8] Feigin VL, Theadom A, Barker-Collo S et al.; BIONIC Study Group. Incidence of traumatic brain injury in New Zealand: a population-based study. Lancet Neurol 2013; 12:53-64.

[9] Harvey LA, Close JC. Traumatic brain injury in older adults: characteristics, causes and consequences. Injury 2012; 43:1821-6.

[10] Thompson HJ, McCormick WC, Kagan SH. Traumatic brain injury in older adults: epidemiology, outcomes, and future implications. J Am Geriatr Soc. 2006; 54:1590-5.

[11] Corrigan JD, Selassie AW, Orman JA. The epidemiology of traumatic brain injury. J Head Trauma Rehabil 2010; 25:72-80.

[12] Fletcher AE, Khalid S, Mallonee S. The epidemiology of severe traumatic braininjury among persons 65 years of age and older in Oklahoma, 1992-2003. Brain Inj 2007; 21:691-9. 